\title{
Transference of natural diversity from the apomictic germplasm of Paspalum notatum to a sexual synthetic population
}

\author{
Alex L. Zilli | Carlos A. Acuña | Roberto R. Schulz | Florencia Marcón | \\ Elsa A. Brugnoli | Sabina F. Novo | Camilo L. Quarin | Eric J. Martínez
}

Instituto de Botánica del Nordeste, Consejo Nacional de Investigaciones Científicas y Técnicas, Facultad de Ciencias Agrarias, Universidad Nacional del Nordeste, Corrientes, Argentina

\section{Correspondence}

Eric J. Martínez, Instituto de Botánica del Nordeste, Consejo Nacional de Investigaciones Científicas y Técnicas, Facultad de Ciencias Agrarias, Universidad Nacional del Nordeste, Sargento Cabral 2131, Corrientes, Argentina. Email: eric@agr.unne.edu.ar

\section{Funding information}

Agencia Nacional de Promoción Científica y Tecnológica and Universidad Nacional del Nordeste, Grant/Award Number: PICTOOTNA 2011-080; Agencia Nacional de Promoción Científica y Tecnológica, Argentina, Grant/Award Number: PICT No 2012-00261; Consejo Nacional de Investigaciones Científicas y Técnicas, Grant/Award Number: PIP 2012-2014 no. 112-201101-00469; Universidad Nacional del Nordeste , Grant/ Award Number: PI A003-2011

\begin{abstract}
Genetic improvement in apomictic forage species has been restricted because of the absence of genetic variability in sexual germplasm with the same ploidy level. Following a new breeding scheme, a sexual synthetic tetraploid population (SSTP) of Paspalum notatum has been generated. The objectives of this work were: (a) to evaluate the genetic variability in SSTP by means of molecular markers, morphologic and agronomic traits, and seed fertility and quality traits and (b) to assess the transference of genetic variability from the apomictic germplasm to the sexual one. Molecular markers revealed a twofold higher level of variability in the SSTP in comparison with the sexual germplasm utilised for its generation, and similar levels with the apomictic ones; moreover, markers showed that most of the variability was inherited from the apomictic germplasm. Morphologic and agronomic traits and seed fertility and quality traits showed high levels of variation in the three groups of genotypes indicating that the new breeding scheme was effective in transferring variability from the apomictic germplasm to the SSTP. This new population will be useful in breeding of $P$. notatum, and the breeding scheme used for its generation may be used in other apomictic species.
\end{abstract}

\section{KEYWORDS}

fertility, genetic improvement, genetic variability, microsatellite markers, sexuality

\section{1 | INTRODUCTION}

Beef cattle production is largely dependent on native or planted forage species. Approximately half the cattle herds of the world is fed with warm-season perennial grasses in tropical and sub-tropical countries, and around $60 \%$ of these grass species reproduce by apomixis (Jank, Valle, \& Resende, 2011). The majority of apomictic forage cultivars are direct selections resulting from exploring the species' natural diversity (Miles, 2007; Vogel \& Burson, 2004). In addition, the large majority of cultivated area is planted with just one genotype, which represents a threat of production losses due to abiotic stresses and pest or disease problems (Jank et al., 2011).

Several studies concerning genetic diversity in populations of apomictic species have demonstrated the existence of a high natural diversity. This can be attributed to gene flow with sexual sympatric populations (Daurelio, Espinoza, Quarin, \& Pessino, 2004; García, Balatti, \& Arturi, 2007; Sartor, Rebozzio, Quarin, \& Espinoza, 2013; Brugnoli et al., 2013, 2014), residual sexuality (Rebozzio, Sartor, Quarin, \& Espinoza, 2011; Sartor et al., 2013) and mutations (Hörandl \& Paun, 2007). Despite that, it is generally accepted that 
apomictic populations are represented by a single or a few genotypes (Brugnoli et al., 2013) due to apomictic reproduction that allows the colonisation of new niches through a single individual (Hörandl, 2010). Therefore, a higher diversity is observed between than within populations (Brugnoli et al., 2013, 2014; Gornall, 1999; GutierrezOzuna, Eguiarte, \& Molina-Freaner, 2009; Paun, Greilhuber, Temsch, \& Hörandl, 2006; Richards, 2003).

Paspalum notatum Flüggé is a warm-season perennial grass native to Central and South America and particularly predominant on the grasslands of southern Brazil, Paraguay, Uruguay and northern Argentina (Gates, Quarin, \& Pedreira, 2004). It is cultivated as forage and utility turf around the world (Blount \& Acuña, 2009). The species has two cytotypes with different modes of reproduction associated. The diploid cytotype ( $2 n=2 x=20$ ) reproduces sexually and is cross-pollinated (Burton, 1955), whereas the tetraploid $(2 n=4 x=40)$ reproduces by aposporous apomixis (Gates et al., 2004). Indigenous diploids inhabit a small area of northeast of Argentina, whereas the tetraploid cytotype grows all over the natural distribution area of the species (Daurelio et al., 2004).

Genetic improvement of diploid P. notatum was carried out mainly by means of recurrent phenotypic selection (Burton, 1982). Several cultivars have been released to the market and a few have been adopted by farmers in the southeastern United States (Blount \& Acuña, 2009). However, the majority of tetraploid cultivars are the result of ecotype selection. After the generation of experimental sexual tetraploid genotypes (ESTG) by chromosome doubling (Burton \& Forbes, 1960; Quarin, Espinoza, Martínez, Pessino, \& Bovo, 2001; Quesenberry, Dampier, Lee, Smith, \& Acuña, 2010), numerous attempts have been made to obtain heterotic apomictic hybrid cultivars through crossing sexual versus apomictic genotypes. However, this breeding scheme has the limitation of low genetic variability of the female counterpart due to difficulties in obtaining sexual tetraploid genotypes. In addition, a low proportion of highly apomictic hybrids have been reported for sexual $x$ apomictic crosses (Acuña, Blount, Quesenberry, Kenworth, \& Hanna, 2009; Zilli et al., 2015). Until the present, the only tetraploid hybrid cultivar of $P$. notatum released to the market is Boyero-UNNE (Reg. No. CV-5, PI 676021) (Urbani et al., 2017). An interesting attempt to increase the available sexual tetraploid germplasm has been made by Quesenberry et al. (2010). These authors created a large number of induced sexual genotypes using different chromosome duplication treatments and tissue culture of the diploid cytotype.

With the hypothesis that the natural diversity can be transferred from the apomictic germplasm of $P$. notatum to a synthetic sexual population, the following three-step procedure was used: (a) crosses were made between three ESTG and 10 natural apomictic tetraploid genotypes (NATG; Zilli et al., 2015); (b) sexual hybrids were identified by molecular markers linked to apospory and (c) a sexual synthetic population was created by intercrossing 29 sexual $F_{1}$ hybrids (Zilli et al., 2018). This population could be of great utility for breeding the species; however, there is insufficient knowledge about the level of genetic variability in comparison with the apomictic wild and sexual experimental germplasms.

The knowledge of genetic variability is the key for planning and implementing breeding programmes (Poehlman, 1995). Many studies have been performed in $P$. notatum assessing genetic variability by means of molecular markers, such as random amplified polymorphic DNA (RAPD; Daurelio et al., 2004), amplified fragment length polymorphism (AFLP; Espinoza, Daurelio, Pessino, Valle, \& Quarin, 2006), intersimple sequence repeat (ISSR; Cidade et al., 2009; Reyno et al., 2012) and simple sequence repeat (SSR; Cidade et al., 2010), and/or through morphologic markers (Barreto, 1974; Canto-Dorow, 1996; Cidade et al., 2013; Fachinetto, Dall'Agnol, Lopes de Souza, Weiler, \& Simioni, 2017; De Moraes Fernandes et al., 1973; Reyno et al., 2012). In all evaluated cases, a high genetic variability was found and morphologic markers allowed the differentiation of botanical groups. However, minimal or no correlation was found between molecular and morphologic variability (Cidade et al., 2013; Reyno et al., 2012).

Seed production and quality are fundamental traits for both the seed industry and farmers (Rios et al., 2015), and lack of either or both can thwart the adoption of new cultivars by farmers (Jank et al., 2011). P. notatum is not the exception because low seed germination levels, due to seed dormancy, have been reported as an important limitation for its adoption as a forage species (West \& Marousky, 1989). However, a high variability for seed set and germination have been observed in $P$. notatum, especially for sexually reproducing genotypes (Acuña, Blount, Quesenberry, Hanna, \& Kenworthy, 2007; Acuña et al., 2009), which would be very useful for breeding programmes, especially taking into account the high heritability reported for seed set and seed emergence in the species (Rios et al., 2017).

Fast germination and rapid growth are key factors for successful seedling establishment in order to reach an appropriate stand in the pasture, allowing optimal use of resources (Moser, 2000). Giordano, Berone, and Tomás (2013) succeeded in improving seedling vigour by indirect selection increasing seed weight. However, the authors did not find a relationship between seed weight and germination rate. Moreover, Tomás, Berone, Pisani, Ribotta, and Biderbost (2007) reported a high correlation between seed weight and germination percentage in Panicum coloratum when seed weight was under $1.34 \mathrm{mg}$ per seed; after that point, an increase in seed weight did not change seed germination. In addition, Kneebone and Cremer (1955) grouped seeds of Panicum virgatum in classes according to their weight and found that heavier seeds achieved greater seed germination, seedling emergence and vigour.

The objectives of this research were: (a) to analyse the genetic variability of a sexual synthetic tetraploid population (SSTP), three ESTG and a geographically diverse group of NATG by means of molecular markers, morphologic and agronomic traits, and seed fertility and quality traits and (b) to assess the transference of genetic variability from the NATG to the SSTP.

\section{2 | MATERIALS AND METHODS}

\section{1 | Plant material}

Three groups of genotypes were used for this research. A group was composed of three ESTG which was not considered a population but rather as a control of known low heterozygosity content. This group 
included a plant generated by chromosome doubling from a diploid plant (C4-4x) (Quarin et al., 2001), a highly sexual white stigma bahiagrass (SWSB, also named Q3664), selected from an advanced hybrid progeny originally obtained by crossing a sexual colchicineinduced tetraploid plant with a natural apomictic tetraploid bahiagrass bearing white stigmata (Burton \& Forbes, 1960), and a sexual plant Q4205 obtained by self-pollination from SWSB (Quarin et al., 2003). The second group was formed by 10 NATG collected along the natural distribution range of the species (Figure 1). Finally, the third group consisted of 306 genotypes belonging to a SSTP (Zilli et al., 2018). This population was created by intercrossing 29 sexual $F_{1}$ hybrids, which were generated by crossing the sexual germplasm named ESTG with the apomictic ecotypes identified as NATG.

\section{2 | Molecular analysis}

The molecular analysis was performed on 3 ESTG, 10 NATG and 306 individuals from the SSTP.

Genomic DNA extraction was performed using $150 \mathrm{mg}$ of apical meristem of each plant and following the protocol described by Brugnoli et al. (2014). DNA integrity and quantity were estimated using known concentration DNA patterns. Electrophoresis was performed in $1 \% \mathrm{wt} / \mathrm{vol}$ agarose gel containing $\times 1$ Tris-acetate-EDTA buffer $(40 \mathrm{mM}$ Tris- $\mathrm{HCl}, 5 \mathrm{mM}$ sodium acetate and $0.77 \mathrm{mM}$ ethylenediaminetetraacetic acid, $\mathrm{pH} 8.0$ ) at $40 \mathrm{~V}$ for $60 \mathrm{~min}$. DNA was stained with ethidium bromide $(1 \mu \mathrm{g} / \mathrm{mL})$ for $30 \mathrm{~min}$, visualised with ultraviolet light, and photographed using a GelDoc-it Imaging System (UVP, LLC). DNA samples were diluted to a concentration of $10 \mathrm{ng} / \mu \mathrm{L}$ for their use in polymerase chain reaction amplification.

Eleven SSR primers of $P$. notatum developed by Cidade et al. (2009) were screened for DNA amplification in a preliminary analysis.

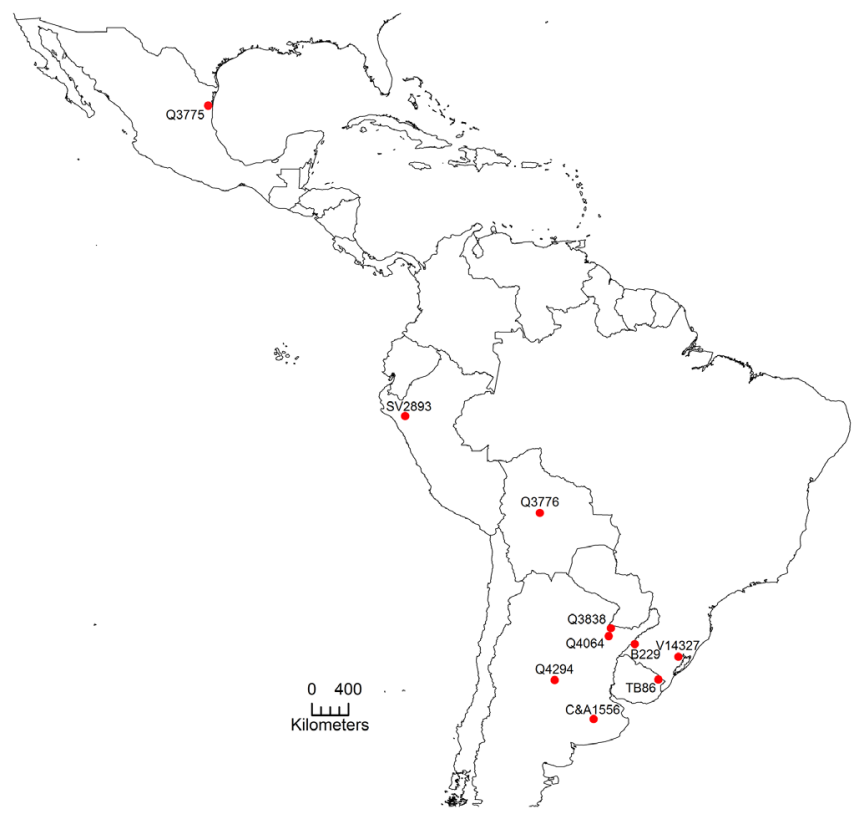

FIGURE 1 Collection sites of the 10 natural apomictic tetraploid genotypes of Paspalum notatum
Finally, seven primers were employed with all genotypes based on the number and intensity of amplified markers, and the percentage of polymorphism observed. DNA amplifications were performed following the protocol described by Cidade et al. (2009) with minor modifications. Polymerase chain reactions were performed in a final volume of $25 \mu \mathrm{L}$ containing $20 \mathrm{ng}$ of genomic DNA, $\times 1$ Taq polymerase reaction buffer, $0.15 \mathrm{mM} 2$ 'deoxinucleósido 5'-trifosfato (dNTPs), $0.8 \mu \mathrm{M}$ of each primer, and $1 \mathrm{U}$ of Taq DNA polymerase (Promega). Amplifications were carried out using a thermal cycler Bio-Rad (My Cycler). Cycles began within $5 \mathrm{~min}$ at $94^{\circ} \mathrm{C}$ followed by 5 cycles of $1 \mathrm{~min}$ at $94^{\circ} \mathrm{C}, 1 \mathrm{~min}$ at $65^{\circ} \mathrm{C}$ (decreasing $1^{\circ} \mathrm{C}$ per cycle) and $1 \mathrm{~min}$ at $72^{\circ} \mathrm{C}$, followed by 25 cycles of $1 \mathrm{~min}$ at $94^{\circ} \mathrm{C}, 1 \mathrm{~min}$ at $60^{\circ} \mathrm{C}$ and $1 \mathrm{~min}$ at $72^{\circ} \mathrm{C}$, and a final extension of $5 \mathrm{~min}$ at $72^{\circ} \mathrm{C}$. Amplicons were separated in $6 \% \mathrm{wt} / \mathrm{vol}$ denaturing polyacrylamide gels, electrophoresed at $300 \mathrm{~W}$ for $3 \mathrm{hr}$, stained using $\times 1$ SYBR Gold nucleic acid gel stain (Invitrogen), and visualised by blue light using an UltraBright LED transilluminator LB-16 (MAESTROGEN). Band patterns were photographed for analysis using a Coolpix L120 (Nikon) camera. The allele scoring was done using the $100 \mathrm{bp}$ DNA Ladder (Promega) as size standards. Fragments with the same molecular size were considered as analogous markers representing the same locus. Due to the fact that the germplasm evaluated was autotetraploid, markers were scored as dominant in a binary matrix with 1 meaning presence and 0 for absence. The molecular analysis was performed using the software Info-Gen developed by the Faculty of Agricultural Science. National University of Cordoba. Argentina. (Balzarini \& Di Rienzo, 2013) and GenAIEx 6.5 developed by the Australian National University (Peakall \& Smouse, 2012).

\section{3 | Analysis of morphologic and agronomic traits}

Plant material for this analysis was a random sample of 140 genotypes from SSTP, together with 3 ESTG and 10 NATG. On September 7,2015 , plants were replicated by rooting tillers in $150-\mathrm{mL}$ seedlings flats and planted into the field 1 month later in 1-m centres. Treatments were arranged in a completely randomised block design with three replications. A border row of $P$. notatum plants was planted around the plot. The experiment was located near to the city of Corrientes, Argentina $\left(27^{\circ} 28^{\prime} \mathrm{S}, 58^{\circ} 47^{\prime} \mathrm{W}\right)$; the soil type was classified as alfic Udipsamment. On November 10, 2015, the plot was fertilised with N-P-K (15-15-15) at a rate of $150 \mathrm{~kg} / \mathrm{ha}$.

Growth habit was estimated by measuring plant height $(\mathrm{PH}, \mathrm{cm})$ and plant diameter (PD, $\mathrm{cm}$ ). $\mathrm{PH}$ was measured from the soil level to the top of the canopy, while PD was determined by an average between the largest and the shortest diameter of a given plant.

Leaf blade length ( $L B L, c m$ ) was measured on the first fully expanded leaf in three different tillers per plant. Leaf blade width (LBW, mm) was also determined by measure at the widest point of the same leaves used for the blade length.

Flowering stem height $(\mathrm{FSH}, \mathrm{cm})$ was determined by an average measure of three stems per plant, measuring from the soil level to the axis of the first raceme of the inflorescence. In the same 
inflorescences, raceme length ( $\mathrm{RL}, \mathrm{cm}$ ) was also determined. All the variables mentioned above were measured on May 1, 2016.

Biomass production per plant (BP, g) was determined on May 25,2016 by cutting plants at $8-\mathrm{cm}$ stubble height. The fresh weight of harvested material was recorded and a subsample per family of halfsibs was collected and dried at $60^{\circ} \mathrm{C}$ for $72 \mathrm{hr}$. After weighing, BP was calculated.

Frost tolerance (FT) was estimated on July 10, 2016, 2 days after the first frost event of the season, when temperatures reached $-2^{\circ} \mathrm{C}$ at soil level, using a 1-to-5 scale, where 1 represents the least frost tolerant and 5 represents the most tolerant plant.

Winter regrowth (WR) was determined on September 23, 2016 using a 1-to-5 scale, where 1 represents plants with the least aboveground growth and 5 represents plants with the greatest amount of biomass.

\subsection{Seed set and quality analysis}

Seed set and quality were estimated on the ESTG, NATG and a random sample of 30 individuals of the SSTP during the year 2017. The evaluated plants were the same cultivated in the plot described above.

Seed set was determined under two different pollination methods, using three replications per plant, during January 2017. Self-pollination seed set was measured by enclosing three to four inflorescences per plant in glassine bags a day prior to the anthesis of spikelets. Openpollination seed set was estimated using three or four inflorescences per plant, enclosing them after anthesis in order to avoid seed loses. Inflorescences were harvested 30 days after anthesis, dried at $37^{\circ} \mathrm{C}$ for $48 \mathrm{hr}$ and manually threshed. A seed blower was used to separate the spikelets with seed (caryopsis) from the empty ones. Each replica was processed separately, and the weight of 1,000 seeds was calculated for each. Germination of seeds was evaluated by sowing three replicas of 50 seeds each on October 19, 2017 under greenhouse conditions. Seeds had a storage period of around 7 months at room temperature. Germination was recorded daily until 21 days after sowing. The speed of germination was calculated based on the following equation: germination at 8 days $\times$ germination at 21 days $/ 100$.

\section{5 | Statistical analysis}

Because plant material used in this study was tetraploid, markers were scored and analysed as dominants. DNA amplification profiles obtained for each plant were introduced into a binary-data matrix. The presence of a marker was scored as 1 and absence as 0 . The resulting binary-data matrix was analysed using a statistical software package, Info-Gen (Balzarini \& Di Rienzo, 2013). Genetic distances among individuals were measured by the Jaccard's dissimilarity coefficient (1-S). Correlation among genetic and geographic distance was estimated in the NATG using Pearson's correlation coefficient.

Morphologic and agronomic traits, and seed set were analysed using Info-Gen software (Balzarini \& Di Rienzo, 2013) as a completely randomised block design with three replications (vegetative clones), whereas seed quality traits were analysed as a completely randomised design with three replicates. Data were analysed using analysis of variance (ANOVA). Fisher's least significant difference (LSD) test was used for comparing pairs of means, using a significance at $p<.05$. Narrow sense heritability $\left(h^{2}\right)$ was calculated by estimation of variance components using the statistical software R 3.4.2 (R Core Team, 2016) by means of the generalised mixed linear models. Correlation among traits was estimated using Spearman's correlation coefficient for morphoagronomic traits and Pearson's correlation coefficient for seed fertility and quality traits. Principal component analysis (PCA) was also performed to estimate distribution of genotypes and group of genotypes.

\section{3 | RESULTS}

\section{1 | Molecular characterisation}

A total of 124 markers were amplified in the SSTP, the NATG and the GTSE using seven SSR primers, with a range from 11 to 35 markers per primer (average of 18). SSRs primers showed a high polymorphism with only two monomorphic markers (average of $98 \%$ of polymorphism) (Table 1). Because markers were scored as dominant, it was not possible to determine allelic frequency. Polymorphic information content (PIC) was calculated to estimate the level of information provided by each primer. The PIC varied from 0.23 to 0.36 , and considering that PIC varied from 0 to 0.5 for dominant markers, these primers can be considered as moderately to highly informative (Table 1).

The SSTP amplified 124 loci, a similar amount was observed in NATG (122), whereas ESTG showed only 68 (Table 2). The higher percentage of polymorphic loci (PLP) was observed in the SSTP (98), followed by NATG (91) and finally by ESTG (39) (Table 2). The expected heterozygosis was similar between SSTP and NATG $(0.31$ and 0.29 , respectively), and lower in the ESTG (0.15) (Table 2).

The genetic distance measured by Jaccard's dissimilarity coefficient varied from 0.45 to 0.59 in the ESTG, with the lesser distances belonging to genotypes Q4205 and SWSB, whereas NATG showed

TAB LE 1 Molecular analysis performed with SSR markers in the sexual synthetic tetraploid population, the natural apomictic tetraploid genotypes and the experimental sexual tetraploid genotypes of Paspalum notatum

\begin{tabular}{|lrrrrl|}
\hline Primer & PM & MM & TM & PPL & PIC \\
\hline PN03-A6 & 16 & 0 & 16 & 100 & 0.27 \\
\hline PN02-G10 & 12 & 1 & 13 & 92 & 0.28 \\
\hline PN03-A5 & 11 & 0 & 11 & 100 & 0.27 \\
\hline PN02-H7 & 16 & 1 & 17 & 94 & 0.24 \\
\hline PN03-G8 & 12 & 0 & 12 & 100 & 0.23 \\
\hline PN03-H10 & 20 & 0 & 20 & 100 & 0.36 \\
\hline PN03-F6A & 35 & 0 & 35 & 100 & 0.34 \\
\hline Total & 122 & 2 & 124 & 98 & 0.28 \\
\hline
\end{tabular}

Abbreviations: MM, monomorphic markers; PIC, polymorphic information content; PM, polymorphic markers; PPL, percentage of polymorphic loci; TM, total markers. 
TAB LE 2 Estimators of genetic variability based on seven simple sequence repeat (SSR) primers for the sexual synthetic tetraploid population (SSTP), the natural apomictic tetraploid genotypes (NATG) and the experimental sexual tetraploid genotypes (ESTG) of Paspalum notatum

\begin{tabular}{llll}
$\begin{array}{l}\text { Group of } \\
\text { genotypes }\end{array}$ & $\begin{array}{l}\text { Total no. } \\
\text { of loci }\end{array}$ & $\begin{array}{l}\text { Percentage of } \\
\text { polymorphic loci }\end{array}$ & $\begin{array}{l}\text { Expected } \\
\text { heterozygosis }\end{array}$ \\
\hline SSTP & 124 & 98 & 0.31 \\
\hline NATG & 122 & 91 & 0.29 \\
\hline ESTG & 68 & 39 & 0.15 \\
\hline
\end{tabular}

range from 0.36 to 0.67 (data not shown). Distances between genotypes of the SSTP varied from 0.02 to 0.82 (data not shown). There was no correlation between molecular and geographic distances for the NATG $(p=.56)$.

In an analysis of private markers, it was observed that the three groups of genotypes shared 56 markers, while 68 markers were amplified differentially for each of them. The SSTP amplified 66 markers present in the NATG which were absent in the ESTG, therefore, these were transferred from the apomictic germplasm. On the other hand, only two markers were shared between the SSTP and ESTG which were absent in the NATG, therefore, these were inherited from the experimental sexual germplasm.

\section{2 | Morphologic and agronomic characterisation}

A series of six morphologic and three agronomic traits were evaluated in the ESTG, NATG, and a sample of 140 genotypes representative of the phenotypic variation from the SSTP.
The SSTP showed a taller PH than the NATG, whereas ESTG did not show significant differences with the other groups. The NATG exhibited greater PD than the remaining groups of genotypes. Finally, the SSTP showed greater long blade length than the other two groups. No significant differences were found for the remaining morphologic traits $(p>.05)$

The biomass production of the ESTG averaged $153 \mathrm{~g}$ per plant (Table 3), with a range from $10.4 \mathrm{~g}$ (genotype C4-4x) to $328 \mathrm{~g}$ (genotype SWSB). The NATG had an average production of $165 \mathrm{~g}$ per plant (Table 3), with genotype TB86 being the least productive (50.4 g) and Q3776 the most productive ( $266 \mathrm{~g})$. The SSTP showed an average dry matter production of $196 \mathrm{~g}$ per plant (Table 3), with a range from $0 \mathrm{~g}$ (genotype J10\#9 had a height of less than $8 \mathrm{~cm}$ ) to $490 \mathrm{~g}$ (genotype H12\#1). No significant differences were found among the three groups of genotypes. Similarly, no significant differences were found among the three groups for FT and WR $(p>.05)$.

The ANOVA revealed that the LBL and LBW traits were significantly affected by "Genotype" only, suggesting a certain degree of independence for environmental conditions. The remainder evaluated traits showed a significant effect for both "Genotype" and "Block" sources of variation, but the interaction "Genotype $\times$ Block" was not significant for any variable $(p>.05)$.

Coefficients of variation (CV) were used to estimate variability in the three groups of genotypes. Agronomic traits showed greater CVs in comparison with the morphologic ones. BP, FT, and WR showed values of $69.0,43.5$ and 37.6 , respectively. Meanwhile, the morphologic traits showed values of 31.0, 25.5, 23.5, 19.7, 16.9 and 14.4 for $\mathrm{PD}, \mathrm{PH}, \mathrm{LBL}, \mathrm{FSH}, \mathrm{RL}$ and LBW, respectively (Table 3 ).

TA B LE 3 Mean, standard error (SE), coefficients of variation (CV) and narrow sense heritability $\left(h^{2}\right)$ for nine morpho-agronomic traits evaluated in the natural apomictic tetraploid genotypes (NATG), experimental sexual tetraploid genotypes (ESTG) and sexual synthetic tetraploid population (SSTP)

\begin{tabular}{|c|c|c|c|c|c|c|c|c|c|}
\hline & $\mathrm{PH}(\mathrm{cm})$ & $\mathrm{PD}(\mathrm{cm})$ & LBL $(\mathrm{cm})$ & LBW $(\mathrm{cm})$ & $\mathrm{FSH}(\mathrm{cm})$ & $\mathrm{RL}(\mathrm{cm})$ & $\mathrm{BP}(\mathrm{g})$ & $\mathrm{FT}^{\mathrm{a}}$ & $W^{a}$ \\
\hline \multicolumn{10}{|l|}{ NATG } \\
\hline Mean & 33.4 & 33.5 & 27.4 & 8.57 & 58.4 & 12.8 & 165 & 2.5 & 2.6 \\
\hline $\mathrm{CV}$ & 31.9 & 17.6 & 23 & 15 & 16.6 & 21.7 & 77.7 & 29.2 & 37.2 \\
\hline SE & 6.42 & 2.74 & 2.79 & 0.39 & 5.73 & 0.77 & 50.8 & 0.26 & 0.34 \\
\hline $\mathrm{CV}$ & 49.3 & 32.5 & 30.7 & 15.6 & 33.1 & 18.2 & 99.7 & 41.4 & 39.7 \\
\hline \multicolumn{10}{|l|}{ SSTP } \\
\hline$h^{2}$ & 0.43 & 0.4 & 0.32 & 0.48 & 0.57 & 0.58 & 0.38 & 0.31 & 0.43 \\
\hline
\end{tabular}

Abbreviations: BP, biomass production; FSH, flowering stem height; FT, frost tolerance; LBL, leaf blade length; LBW, leaf blade width; PD, plant diameter; $\mathrm{PH}$, plant height; RL, raceme length; WR, winter regrowth.

aVisually estimated using a 1-to-5 scale. 
The comparison between the $\mathrm{CV}$ of the different group of genotypes was variable depending on the trait considered. For instance, a higher CV was observed for ESTG for BP, WR, PH and PD, LBL and LBW and FSH. The NATG had a greater CV for RL, whereas the SSTP showed a greater CV for FT (Table 3).

Narrow sense heritability $\left(h^{2}\right)$ varied between 0.31 and 0.58 . The higher $h^{2}$ values were found in the traits FSH and RL 0.57 and 0.58 , respectively), whereas the lowest values were observed in the traits FT and LBL (0.31 and 0.32, respectively).

Principal component analyses grouped seven NATG in the positive sector of Principal Component 2 (PC2), characterised mainly by greater PD. The ESTG were distributed without following a defined pattern, for instance, genotype C4-4x was located at the negative sector of PC1, characterised by low vigour, whereas SWSB was at the positive sector exhibiting greater plant vigour. The 140 genotypes from the SSTP showed a wide distribution; even out of the distribution area of the NATG and ESTG throughout the PC1, which represented 53.5\% of the variation. Considering the area of PCA covered by each group of genotypes, it was possible to observe that SSTP covered a much wider area than the other groups suggesting a greater variation for the evaluated traits (Figure 2).

The analysis of Spearman's correlation did not show a negative correlation among traits. All agronomic traits were highly correlated $(r>.64 ; p<.001)$. On the other hand, morphologic traits were less correlated among themselves. $\mathrm{PH}$ was correlated with $\mathrm{LBL}(r=.61$; $p<.001)$ and FSH $(r=.59 ; p<.001)$, whereas FSH was correlated with $\mathrm{RL}(r=.56 ; p<.001)$. A correlation between agronomic and morphologic traits was found for BP with FSH $(r=.51 ; p<.001)$ and $\mathrm{PH}$ $(r=.61 ; p<.001)$, and between PH and WR $(r=.56 ; p<.001)$.

\section{3 | Seed set and quality}

Seed set, the weight of 1,000 seeds (W1000), germination percentage at 21 days after sowing (Germ.) and speed of germination (S.G.) were estimated in the three groups of genotypes.

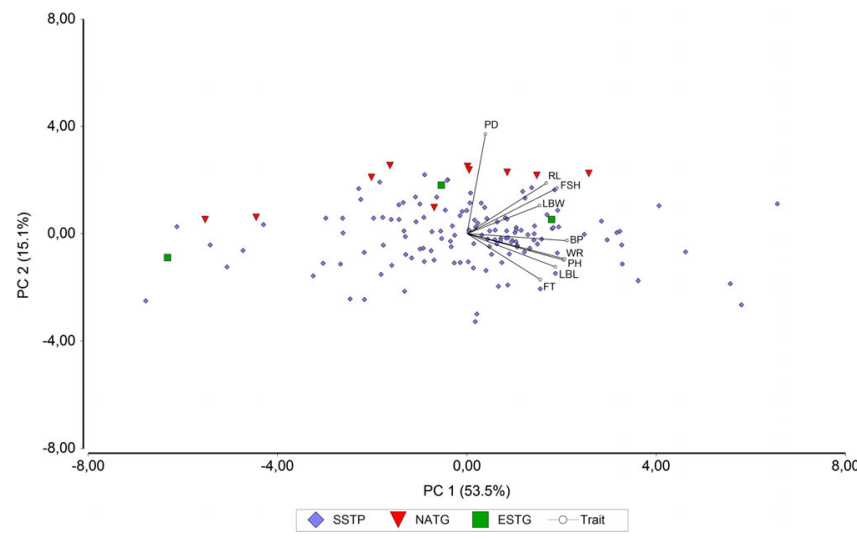

FIGURE 2 Principal component analysis based on nine morphological and agronomic traits. Plant diameter (PD), plant height $(\mathrm{PH})$, leaf blade length (LBL), leaf blade width (LBW), flowering stem height $(\mathrm{FSH})$, raceme length ( $\mathrm{RL})$, biomass production (BP), frost tolerance (FT) and winter regrowth (WR)
There was no interaction between genotype and pollination method for any of the four evaluated variables.

Significant differences were found for seed set among the three groups of genotypes for both pollination methods. Seed set in selfpollinated plants was greater in NATG (18.1\%) than in the ESTG and SSTP (7.4 and 10.3\%, respectively). Similarly, seed set under openpollination was greater in NATG (32.1\%), followed by SSTP $(23.4 \%)$, and finally by ESTG (10.9\%) (Table 4). Moreover, seed set in NATG and SSTP was greater under open-pollination than self-pollination, whereas non-significant differences were found for ESTG between both pollination methods ( $p>.05$ ).

The W1000 showed a mean of 3.42 and $3.44 \mathrm{~g}$ for self- and open-pollination, respectively, without significant differences between them $(p=.99)$. The ANOVA considering both pollination methods together showed that NATG and SSTP had greater W1000 (3.64 and $3.54 \mathrm{~g}$, respectively) than ESTG (3.13 g).

No significant differences were found for germination of seeds among the three groups of genotypes $(p=.68)$ for either pollination method $(p=.13)$. The overall mean of percentages of germination observed in this work was $29.9 \%$.

There were no significant differences for the speed of germination between pollination methods ( $p=.23$ ). Similarly, there were no differences among groups of genotypes when the ANOVA was performed including both pollination methods $(p=.15)$. The overall mean for the speed of germination was $7.24 \%$ (Table 4).

The CVs for self-pollination were greater in ESTG for most of the seed quality traits, with the only exception of S.G. where the SSTP was greatest. When the comparison was performed for variables under openpollination, the ESTG showed the greatest CV for percentage of seed set (S.s.) and W1000, followed by NATG in S.s. and by SSTP in W1000. The NATG showed the greatest value for Germ. Finally, just like under selfpollination, the SSTP showed the greatest CV for S.G. (Table 4).

Narrow sense heritability showed the greatest values for Germ. and W1000 in both pollination methods (Table 4). The lowest $h^{2}$ values were found for S.G. (0.25) and S.s. (0.36) under open- and selfpollination, respectively. The $h^{2}$ values for seed set were almost the same under both pollination methods (Table 4).

Using PCA, based on open-pollinated data, it was possible to observe that the ESTG grouped at the negative sector of PC2 characterised mainly by low seed set (Figure 3). In addition, just like in the morpho-agronomic evaluation, genotypes C4-4x and SWSB showed a clear contrast of performance. While C4-4x exhibited very low performance for all evaluated seed set and quality traits, SWSB showed superior performance (with exception of seed set). The NATG did not form a well-defined group, but almost all of them were located in the positive sector of PC2, characterised by greater seed set. The ESTG and NATG showed a wide distribution across PC1, however, the majority of them are located in the negative sector, characterised by low W1000, Germ. and S.G. (Figure 3). Finally, the individuals of the SSTP showed a wide distribution across the four quadrants of the PCA indicating a broad variation for all the evaluated seed quality traits (Figure 3). However, the majority of them are located at the negative sector of PC2 (Figure 3). 
TA B LE 4 Mean, standard error (SE), coefficients of variation (CV) and narrow sense heritability $\left(h^{2}\right)$ for seed quality traits evaluated in the natural apomictic tetraploid genotypes (NATG), experimental sexual tetraploid genotypes (ESTG) and sexual synthetic tetraploid population (SSTP)

\begin{tabular}{|c|c|c|c|c|c|c|c|c|}
\hline & \multicolumn{4}{|c|}{ Self-pollination } & \multicolumn{4}{|c|}{ Open-pollination } \\
\hline \multicolumn{9}{|l|}{ NATG } \\
\hline Mean & 18.1 & 3.57 & 34.9 & 9.54 & 32.1 & 3.5 & 27.1 & 5.62 \\
\hline SE & 1.74 & 0.13 & 4.03 & 2.35 & 1.76 & 0.11 & 4.12 & 1.95 \\
\hline $\mathrm{CV}$ & 51.9 & 20.3 & 62.2 & 137 & 29.0 & 17.2 & 83.3 & 164 \\
\hline \multicolumn{9}{|l|}{ ESTG } \\
\hline Mean & 7.4 & 3.07 & 30 & 10.8 & 10.9 & 3.19 & 30.6 & 5.60 \\
\hline$S E$ & 3.48 & 0.25 & 12.41 & 6.55 & 3.93 & 0.27 & 8.29 & 2.52 \\
\hline CV & 124 & 22.4 & 109 & 182 & 95.7 & 22.6 & 76.5 & 135 \\
\hline \multicolumn{9}{|l|}{ SSTP } \\
\hline $\mathrm{CV}$ & 102 & 18.8 & 78.4 & 195 & 46 & 15.8 & 72.3 & 188 \\
\hline$h^{2}$ & 0.36 & 0.52 & 0.58 & 0.42 & 0.38 & 0.43 & 0.46 & 0.25 \\
\hline
\end{tabular}

Abbreviations: S.s., seed set; W1000, weight of 1,000 seeds; Germ., germination percentage; S.G., speed of germination.

An analysis of Pearson's correlation was performed for all evaluated genotypes under open-pollination. A high correlation was found for germination and speed of germination $(r=.84 ; p<.001)$. A significant correlation was also found between germination and the W1000 $(r=.44 ; p<.001)$, and between the speed of germination and the $\mathrm{W} 1000(r=.3 ; p<.001)$.

\section{4 | DISCUSSION}

Genetic variability is the key for planning and implementing breeding programmes, allowing a better utilisation of resources and increasing the improvement efficiency (Poehlman, 1995). Recently our research group has generated a sexual synthetic population of $P$. notatum, attempting to transfer the genetic variability from the apomictic to the sexual tetraploid germplasm (Zilli et al., 2018). This population is expected to be the base gene pool for breeding of tetraploid $P$. notatum. However, the genetic variability included in this novel germplasm is unknown. In this work, we performed a wide genetic characterisation of the SSTP in order to know its diversity and the efficiency of the breeding scheme used to transfer the variability from the apomictic to the sexual germplasm.

The seven SSR primers amplified numerous markers with a high level of polymorphism. The PIC was variable depending on the primer,
FIGURE 3 Principal component analysis based on the four seed quality traits obtained under open-pollination conditions. Percentage of seed set (S.s.), weight of 1,000 seeds (W1000), germination of seeds (Germ.) and speed of germination (S.G.)

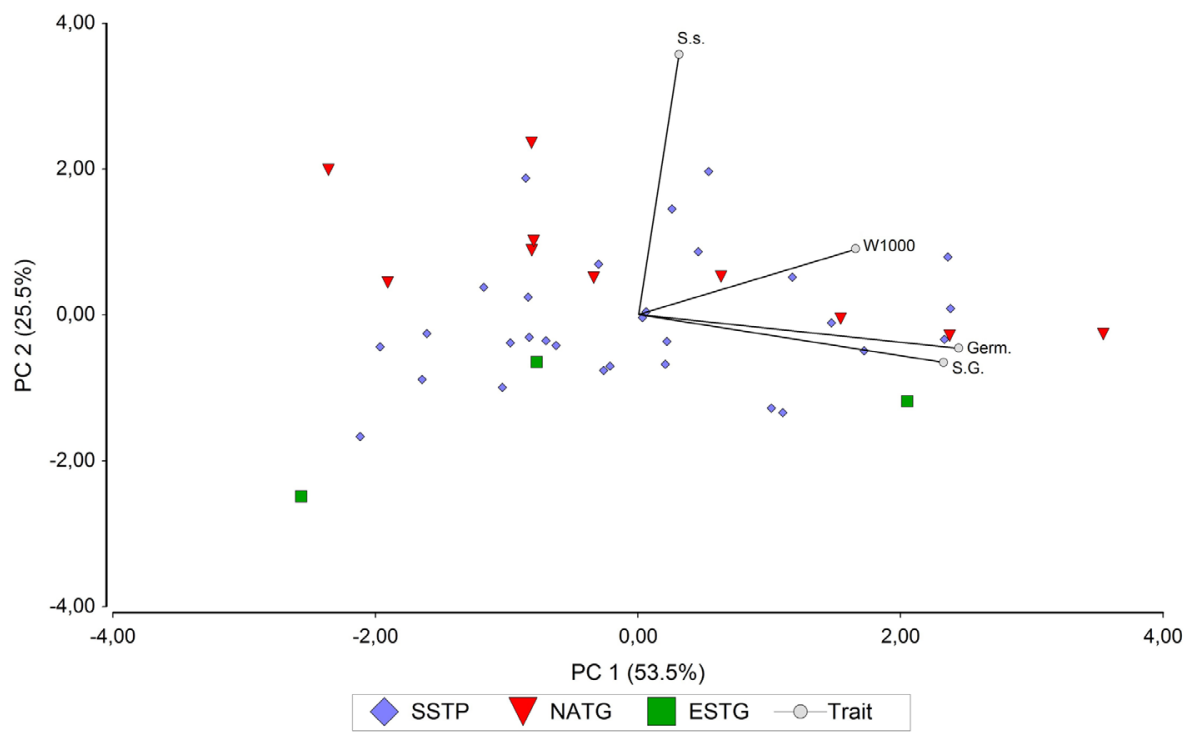


and, taking into account that it was used as dominant, the obtained data can be considered moderately to highly informative. The PICs reported in this study were lower than those reported by Cidade et al. (2009) using the same primers. However, the ranking of primers according to their PIC was the same. In addition, the average markers amplified per primer in this work were twofold greater than those reported by Cidade et al. (2009), which may be due to differences on the stain method or quality of the DNA-Polymerase enzyme. The genetic distances found between the 10 NATG used to generate the SSTP were large and greater than those reported by Espinoza et al. (2006) using AFLP markers and by Cidade, Dall'agnol, Bered, and Souza-Chies (2008) using ISSR markers. These differences may be due, on one hand, to the diverse geographic origin of the genotypes used in this work and on the other hand, to the high variation of microsatellites on the genome as a consequence of a high mutation rate (Toutz, 1989).

The lack of correlation between molecular and geographic distances reported for the NATG shows the absence of a geographic structure of the apomictic germplasm used in this study. This lack of structure could be the result of colonisation of new ecologic niches from one or a few apomictic individuals. The lack of correlation between molecular and geographic distances has been reported previously in P. notatum by Cidade et al. (2008) and Reyno et al. (2012), in P. simplex by Brugnoli et al. (2014), and similar results, were also reported for other apomictic species (Paun et al., 2006). In addition, considering that diploid cytotype is the key for the origin of the tetraploid one (Ortiz et al., 2013), it is probable that the natural distribution of the diploid cytotype was wider than that observed in the present.

The percentage of polymorphic loci found in the SSTP was slightly greater than that observed in the NATG, and 2.5-fold greater than that observed in the ESTG. Similarly, the expected heterozygosis of the SSTP was comparable to the NATG and much greater than the ESTG. The SSTP amplified 56 markers unique to NATG and only two unique to ESTG. This result is a consequence of a greater number of allelic variants contributed by NATG than by ESTG. The ESTG group was composed by only three genotypes and two of them were closely related since Q4205 was derived from SWSB by self-pollination in a sexual reproductive event (Quarin et al., 2003). On the other hand, the sexual plant $C 4-4 x$ is a doubled diploid with an allelic set up duplex for all loci; this means that its heterozygosity content is lower with respect to a hybrid or open pollinated (auto)tetraploid. The SSTP amplified the 122 markers presents in the NATG showing that the natural variability of apomictic germplasm was effectively transferred to the sexual synthetic tetraploid germplasm.

There were significant differences among the three groups of genotypes for the morphological traits LBL and LBW, and PH and PD, whereas the remaining traits did not show differences. The relationship between PH and PD indicate the growth habit of plants. The SSTP showed an average PH that was twofold greater than the diameter (44.8 and $22.6 \mathrm{~cm}$, respectively), which indicates an erect growth habit. The NATG showed similar PH and PD $(33.4$ and $33.5 \mathrm{~cm}$, respectively) indicating a prostrate growth habit. Finally, the ESTG showed a 1.6-fold larger PH than PD (39.1 and $25.4 \mathrm{~cm}$, respectively), which is associated with a semi-erect growth habit. Moreover, the CVs for growth habit were similar among the three groups of genotypes (33, 38 and 39\% for NATG, ESTG and SSTP, respectively; data not shown), indicating that natural variability for this trait present in NATG and ESTG was transferred to the SSTP. A prostrate growth habit in $P$. notatum has a relation with the persistence of the pasture under grazing and the ability to cover the soil and compete with weeds, whereas an erect growth habit is more related to higher BP (Acuña, Blount, Quesenberry, Kenworthy, \& Hanna, 2011; Acuña et al., 2009; Interrante et al., 2009). Fortunately, the high CV observed for PH and PD in the SSTP, and the high heritability and the lack of negative correlation between both traits, show that it would be possible to select plants that combine persistence and high forage production.

The lack of correlation between molecular versus morphologic and agronomic variability could be due to the type of molecular marker employed. Microsatellites are usually located on non-coding regions of the genome; therefore, genes involved in the expression of the evaluated traits were apparently not associated with the microsatellites used on this work. This lack of correlation was also observed using ISSR markers (Cidade et al., 2013; Reyno et al., 2012).

The CVs among the three groups of genotypes varied depending on the evaluated trait, unlike what was observed in the molecular analysis. In general, there were similar levels of variability between the SSTP and NATG for both the comparison of CVs and the PCA. This finding is clear evidence that the variability of NATG was transferred to the SSTP. In addition, the high CV observed in the ESTG, particularly for agronomic traits, is attributed to the huge differences in vigour and agronomic performance among genotypes included in this group. The genotype $C 4-4 x$ is an autotetraploid plant generated by chromosome duplication with colchicine from a diploid plant, characterised by very low vigour, whereas the genotype SWSB has a hybrid origin, and it was selected based on its high vigour and agronomic performance. The relatively high homozygosity is known to be negatively correlated with vigour in autotetraploid seed propagated plants. The highest yield is expected when alleles correlated with vigour are heterozygous and in trans chromosome conditions (theory of linkats; Demarly, 1979).

The PCA revealed a wide distribution of the 140 individuals of the SSTP, even outside of the range covered by its ancestors, ESTG and NATG. This result indicated that the SSTP has individuals with a new and useful combination of traits. In Figure 2, it can be seen that around $10 \%$ of the SSTP combined several traits of interest for genetic improvement. The lack of negative correlation between traits, and the fact that many of these traits (particularly, the agronomic ones) are positively correlated, is a clear advantage for using this synthetic population for breeding programmes. The intermediate levels of narrow sense heritability reported in this work suggest that recurrent selection based on specific or general combining ability (Comstock, Robinson, \& Harvey, 1949) would be suitable for breeding this germplasm. In addition, the hybrids obtained in crosses performed with apomictic genotypes could be evaluated as potential new cultivars, and at the same time improving the synthetic population (Miles, 
2007). Another option might be the use of recurrent restricted phenotypic selection (Burton, 1982), which was successful in breeding the diploid germplasm of the species (Blount \& Acuña, 2009).

Seed set under self-pollination was greater in the NATG than that observed in sexual tetraploid genotypes. This result is expected for apomictic plants (Vogel \& Burson, 2004) and is in agreement with previous reports for the species (Acuña et al., 2007; Burton, 1948). The NATG showed twofold greater open- than self-fertility, similar to the observed levels in the SSTP. Nevertheless, the NATG exhibited greater open-fertility than the SSTP, whereas the ESTG showed the lowest value. Marked variation was observed among genotypes on the three evaluated groups under open-pollination, an even greater level than under self-pollination. The higher CV observed for the ESTG for both self- and open-fertility is due to the contrasting genotypes included in this group, as explained above. The CV of the SSTP under both pollination methods was greater than that observed on the NATG, indicating a greater variability for these traits in the sexual germplasm. In addition, the observed variation on the SSTP would be useful for selecting highly self-sterile plants for production of hybrids, and at the same time, highly cross-fertile plants to improve seed production.

There were no significant differences either for seed quality traits or for pollination methods among the three groups of genotypes. The average seed weight reported for the three analysed groups in this work (3.58 g) was greater than that reported by Lopes et al. (2011) for apomictic tetraploid entries $(2.27 \mathrm{~g}$ ). In turn, the average seed weight in the three groups of genotypes was similar to those reported by Rios et al. (2015) for the apomictic tetraploid cultivar Argentine ( $\approx 3.6 \mathrm{~g}$ ). Seed weight showed the least variation among the seed quality traits evaluated in this study. The CVs for this trait were similar between the three groups of genotypes, supporting the hypothesis of effective transference of variability from apomictic genotypes to the SSTP.

Seed germination in P. notatum, as in almost all warm-season grasses, has been considered the main factor affecting success in establishment, together with seedling vigour (West \& Marousky, 1989; Williams \& Webb, 1958). Low seed germination in $P$. notatum is related to dormancy in viable seeds, due to the presence of coat factors (West \& Marousky, 1989). Seed dormancy depends on genotypes evaluated and environmental conditions during seed production and seed filling (Adjei, Mislevy, \& Chason, 1992; Gates \& Burton, 1998). The average seed germination observed in this study was $29 \%$ after an aging period of approximately 7 months. High variability was observed in the evaluated germplasm for this trait, indicating that improvement would be possible in this plant material.

Rapid germination and emergence of seedlings are key factors for successful establishment of the pasture (West \& Marousky, 1989). The overall rate of germination observed in this work was $6 \%$, with no significant differences among the three groups of genotypes or between pollination methods. Gates and Burton (1998) reported 16.4 and $4.7 \%$ of seedling emergence 7 days after sowing for years 1995 and 1996, respectively. They observed differences between entries of diploid $P$. notatum that favoured the improved germplasm, and this was because one of the breeding targets was seedling vigour, obtained by early germination. Giordano et al. (2013) reported an improvement in seedling vigour and seedling emergence 7 days after sowing, after 1 cycle of selection targeting greater seed weight in $P$. coloratum var. makarikariense, but they found no differences in seedling emergence 14 days or more after sowing. The high heritability observed for seed weight and germination, and the correlation observed between both traits, is a useful finding for breeding the species. The selection over an easily measured trait, such as seed weight, would likely also result in improved seed germination and speed of germination. The correlations observed in this work have been observed previously for this species by Adjei et al. (1992) and Gates and Burton (1998).

CV for seed set and quality traits were relatively high in the ESTG despite the low number of individuals in this group. This finding may be related to the large differences in vigour and fertility between genotypes C4-4x and SWSB, due to their origin, as was explained above. Sexual germplasm of $P$. notatum (ESTG and SSTP) showed greater variability for seed set than NATG. CVs were similar for seed weight and rate of germination among the groups of genotypes for both pollination methods, as well as for seed germination under open pollination. This finding indicates that there was not a reduction in variability during the generation of the SSTP. Finally, the PCA showed a wide distribution of the individuals from the SSTP for both principal components, which indicates the presence of a new combination of traits in this novel sexual tetraploid germplasm. The high variability observed in this novel population together with the moderate to high heritability observed indicate that it would be possible to improve this population using breeding methods developed for cross-pollinated species such as the recurrent restricted phenotypic selection developed by Burton (1982). In these cases, it would be important to select highly self-sterile sexual plants to avoid inbreeding. Further studies should be performed on this germplasm in order to identify individuals of high general and/or specific combining ability on crosses with elite apomictic cultivars, to be included in a recurrent selection scheme aimed at accumulating additive and non-additive effects.

In conclusion, the molecular analysis performed with microsatellite markers indicated that the SSTP presented an average of twofold greater variability than ESTG, and similar levels to NATG. Most polymorphic markers (98\%) present in SSTP were transferred from NATG. Moreover, the variability observed in SSTP for a series of morphological and agronomic traits and seed fertility and quality traits was as high as that present in NATG. These findings indicate that the variability inherent in a group of natural apomictic genotypes, collected from diverse origins, was effectively transferred to the novel sexual synthetic tetraploid germplasm of $P$. notatum, providing a valuable genetic resource for breeding programmes with this species.

\section{ACKNOWLEDGEMENTS}

We thank Dr. Lynn E. Sollenberger, University of Florida, for reviewing this manuscript before submission. This work was supported by the Agencia Nacional de Promoción Científica y Tecnológica, Argentina (PICT No. 2012-00261); Agencia Nacional de 
Promoción Científica y Tecnológica and Universidad Nacional del Nordeste (PICTO-OTNA 2011-080); Consejo Nacional de Investigaciones Científicas y Técnicas (PIP 2012-2014 No. 112-201101-00469) and Universidad Nacional del Nordeste (PI A003-2011). A.L.Z. and F.M. received a fellowship from CONICET. R.R.S. received a fellowship from UNNE. C.A.A., E.A.B., E.J.M. and C.L.Q. are career members of CONICET.

\section{REFERENCES}

Acuña, C. A., Blount, A. R., Quesenberry, K. H., Hanna, W. W., \& Kenworthy, K. E. (2007). Reproductive characterization of Bahiagrass germplasm. Crop Science, 47, 1711-1717.

Acuña, C. A., Blount, A. R., Quesenberry, K. H., Kenworth, K. E., \& Hanna, W. W. (2009). Bahiagrass tetraploid germplasm: Reproductive and agronomic characterization of segregating progeny. Crop Science, 49, 581-588.

Acuña, C. A., Blount, A. R., Quesenberry, K. H., Kenworthy, K. E., \& Hanna, W. W. (2011). Tetraploid bahiagrass hybrids: Breeding technique, genetic variability and proportion of heterotic hybrids. Euphytica, 179, 227-235.

Adjei, M. B., Mislevy, P., \& Chason, W. (1992). Seed yield of bahiagrass in response to sward management by phenology. Agronomy Journal, 82, 599-603.

Balzarini, M. G., \& Di Rienzo, J. A. (2013). Info-Gen versión 2013. FCA, Universidad Nacional de Córdoba, Argentina. Retrieved from http:// www.info-gen.com.ar

Barreto, I. L. (1974). O gênero Paspalum (Gramineae) no Rio Grande do Sul. (PhD thesis). Rio Grande do Sul, Universidade Federal, Porto Alegre, Brazil.

Blount, A. R., \& Acuña, C. A. (2009). Bahiagrass. In R. J. Singh (Ed.), Genetic resources, chromosome engineering, and crop improvement series: Forage crops (Vol. 5, pp. 81-101). Boca Raton, FL: CRC Press.

Brugnoli, E. A., Urbani, M. H., Quarin, C. L., Martínez, E. J., \& Acuña, C. A. (2013). Diversity in diploid, tetraploid and mixed diploid-tetraploid populations of Paspalum simplex. Crop Science, 53, 1509-1516.

Brugnoli, E. A., Urbani, M. H., Quarin, C. L., Zilli, A. L., Martínez, E. J., \& Acuña, C. A. (2014). Diversity in apomictic populations of Paspalum simplex Morong. Crop Science, 54, 1656-1664.

Burton, G. W. (1948). Artificial fog facilitates Paspalum emasculation. Journal of the American Society of Agronomy, 40, 281-282.

Burton, G. W. (1955). Breeding Pensacola bahiagrass, Paspalum notatum: Method of reproduction. Agronomy Journal, 47, 311-314.

Burton, G. W. (1982). Recurrent restricted phenotypic selection increases bahiagrass forage yield. Crop Science, 22, 109-111.

Burton, G. W., \& Forbes, I. (1960). The genetics and manipulation of obligate apomixis in common bahiagrass (Paspalum notatum Flügge). In C. L. Skidmore, et al. (Eds.), Proceedings of the Eighth International Grassland Congress, Reading, England, July 11-21 (pp. 66-71). Oxford, England: Alden Press.

Canto-Dorow, T. S. (1996). Revisão taxonômica das espécies de Paspalum grupo Notata (Poaceae-Paniceae) do Rio Grande do Sul, Brasil. Iheringia, 47, 3-44.

Cidade, F. W., Dall'agnol, M., Bered, F., \& Souza-Chies, T. T. (2008). Isolation and characterization of microsatellite loci in Paspalum notatum Flüggé (Poaceae). Genetic Resources and Crop Evolution, 55, 235-246.

Cidade, F. W., Souza-Chies, T. T., Batista, L. A. R., Dall'Agnol, M., Zucchi, M. I., Jungmann, L., \& Souza, A. P. (2009). Isolation and characterization of microsatellite loci in Paspalum notatum Flüggé (Poaceae). Conservation Genetics, 10, 1977-1980.

Cidade, F. W., Souza-Chies, T. T., Souza, F. H. D., Batista, L. A. R., Dall'Agnol, M., Valls, J. F. M., ... Souza, A. P. (2010). Microsatellite loci for Paspalum atratum (Poaceae) and cross-amplification in other species. American Journal of Botany, 97, e107-e110.

Cidade, F. W., Vigna, B. B. Z., de Souza, F. H. D., Valls, J. F. M., Dall'Agnol, M., Zucchi, M. I., ... Souza, A. P. (2013). Genetic variation in polyploid forage grass: Assessing the molecular genetic variability in the Paspalum genus. BMC Genetics, 14, 50.

Comstock, R. E., Robinson, H. F., \& Harvey, P. H. (1949). A breeding procedure designed to make maximum use of both general and specific combining ability. Agronomy Journal, 41, 360-367.

Daurelio, L. D., Espinoza, F., Quarin, C. L., \& Pessino, S. C. (2004). Genetic diversity in sexual diploid and apomictic tetraploid populations of Paspalum notatum situated in sympatry or allopatry. Plant Systematics and Evolution, 244, 189-199.

De Moraes Fernandes, M. I., Barreto, I. L., \& Salzano, F. M. (1973). Cytogenetic, ecologic and morphologic studies in Brazilian forms of Paspalum notatum. Canadian Journal of Genetics and Cytology, 15, 523-531.

Demarly, Y. (1979). The concept of linkat. In A. C. Zeven \& A. M. Harten (Eds.), Proceedings of the Conference on Broadening the Genetic Base of Crops (pp. 257-265). Wageningen, the Netherlands: Centre for Agricultural Publishing and Documentation.

Espinoza, F., Daurelio, L. D., Pessino, S. C., Valle, E. M., \& Quarin, C. L. (2006). Genetic characterization of Paspalum notatum accessions by AFLP markers. Plant Systematics and Evolution, 258, 147-159.

Fachinetto, J. M., Dall'Agnol, M., Lopes de Souza, C. H., Weiler, R. L., \& Simioni, C. (2017). Genetic diversity of a Paspalum notatum Flügge germplasm collection. Revista Brasileira de Zootecnia, 46, 714-721.

García, M. V., Balatti, P. A., \& Arturi, M. J. (2007). Genetic variability in natural populations of Paspalum dilatatum Poir. analyzed by means of morphological traits and molecular markers. Genetic Resources and Crop Evolution, 54, 935-946.

Gates, R. N., \& Burton, G. W. (1998). Seed yield and seed quality response of Pensacola and improved bahiagrasses to fertilization. Agronomy Journal, 90, 607-611.

Gates, R. N., Quarin, C. L., \& Pedreira, C. G. S. (2004). Bahiagrass. In L. E. Moser, et al. (Eds.), Warm-season (C4) grasses, Agronomy Monography 45 (pp. 651-680). Madison, WI: ASA, CSSA, SSSA.

Giordano, M. C., Berone, G. D., \& Tomás, M. A. (2013). Selection by seed weight improves traits related to seedling establishment in Panicum coloratum L. var. makarikariense. Plant Breeding, 132, 620-624.

Gornall, R. J. (1999). Population genetic structure in agamospermous plants. In P. M. Hollingsworth, R. M. Bateman, \& R. J. Gornall (Eds.), Molecular systematics and plant evolution (pp. 118-138). London, England: Taylor \& Francis.

Gutierrez-Ozuna, R., Eguiarte, L. E., \& Molina-Freaner, F. (2009). Genotypic diversity among pasture and roadside populations of the invasive buffelgrass (Pennisetum ciliare L. Link) in north-western Mexico. Journal of Arid Environments, 73, 26-32.

Hörandl, E. (2010). The evolution of self-fertility in apomictic plants. Sexual Plant Reproduction, 23, 73-86.

Hörandl, E., \& Paun, O. (2007). Patterns and sources of genetic diversity in apomictic plants: Implications for evolutionary potentials. In E. Hörandl, U. Grossniklaus, P. J. van Dijk, \& T. F. Sharbel (Eds.), Apomixis: Evolutions, mechanisms and perspectives (pp. 170-194). Rugell, Liechtenstein: ARG Gantner Verlag.

Interrante, S. M., Sollenberger, L. E., Blount, A. R., Coleman, S. W., White, U. R., \& Liu, K. (2009). Defoliation management of bahiagrass germplasm affects cover and persistence-related responses. Agronomy Journal, 101, 1381-1387.

Jank, L., Valle, C. B., \& Resende, R. M. S. (2011). Breeding tropical forages. Crop Breeding and Applied Biotechnology, 1, 27-34.

Kneebone, W. R., \& Cremer, C. L. (1955). The relationship of seed size to seedling vigor in some native grass species. Agronomy Journal, 47, 472-477.

Lopes, R. R., Lopes de Souza, C. H., Pereira, E. A., Gasparetto, B. F., Dall'Agnol, M., \& Franke, L. B. (2011). Genetic variability of the 
components of seed yield in interspecific hybrids of Paspalum. Revista Brasileira de Zootecnia, 46, 296-302.

Miles, J. W. (2007). Apomixis for cultivar development in tropical forage grasses. Crop Science, 47, 238-249.

Moser, L. E. (2000). Morphology of germinating and emerging warmseason grass seedlings. In K. J. Moore \& B. E. Anderson (Eds.), Native Warm-Season Grasses: Research Trends and Issues CSSA Special Publication No. 30 (pp. 35-47). Madison, WI: American Society of Agronomy/Crop Science Society of America.

Ortiz, J. P. A., Quarin, C. L., Pessino, S. C., Acuña, C. A., Martínez, E. J., Espinoza, F., ... Pupilli, F. (2013). Harnessing apomictic reproduction in grasses: What we have learnt from Paspalum. Annals of Botany, 112, 767-787.

Paun, O., Greilhuber, J., Temsch, E., \& Hörandl, E. (2006). Patterns, sources and ecological implications of clonal diversity in apomictic Ranunculus carpaticola (Ranunculus auricomus complex, Ranunculaceae). Molecular Ecology, 15, 897-910.

Peakall, R., \& Smouse, P. E. (2012). GenAlEx 6.5: Genetic analysis in Excel. Population genetic software for teaching and research-an update. Bioinformatics, 28, 2537-2539.

Poehlman, J. M. (1995). Germplasm resources and conservation. In J. J. Poehlman \& D. A. Sleper (Eds.), Breeding field crops (pp. 171-186). Ames, IA: lowa State University Press.

Quarin, C. L., Espinoza, F., Martínez, E. J., Pessino, S. C., \& Bovo, O. A. (2001). A rise of ploidy level induces the expression of apomixis in Paspalum notatum. Sexual Plant Reproduction, 13, 243-249.

Quarin, C. L., Urbani, M. H., Blount, A. R., Martinez, E. J., Hack, C. M., Burton, G. W., \& Quesenberry, K. H. (2003). Registration of Q4188 and Q4205, sexual tetraploid germplasm lines of bahiagrass. Crop Science, 43, 745-746.

Quesenberry, K. H., Dampier, J. M., Lee, Y. Y., Smith, R. L., \& Acuña, C. A. (2010). Doubling the chromosome number of bahiagrass via tissue culture. Euphytica, 175, 43-50.

R Core Team. (2016). R: A language and environment for statistical computing. Vienna, Austria: R Foundation for Statistical Computing. Retrieved from https://www.R-project.org/

Rebozzio, R. N., Sartor, M. E., Quarin, C. L., \& Espinoza, F. (2011). Residual sexuality and its seasonal variation in natural apomictic Paspalum notatum accessions. Biologia Plantarum, 55, 391-395.

Reyno, R., Narancio, R., Speranza, P., do Canto, J., López Carro, B., Hernández, P., ... Dalla Rizza, M. (2012). Molecular and cytogenetic characterization of a collection of bahiagrass (Paspalum notatum Flügge) native to Uruguay. Genetic Resources and Crop Evolution, 59, 1823-1832.

Richards, A. J. (2003). Apomixis in flowering plants: An overview. Philosophical Transactions of the Royal Society B, 358, 1085-1093.

Rios, E. F., Blount, A., Harmon, P., Mackowiak, C., Kenworthy, K., \& Quesenberry, K. (2015). Ergot resistant tetraploid bahiagrass and fungicide effects on seed yield and quality. Plant Health Progress, 2(16), 56-62.

Rios, E. F., Kenworthy, K., Blount, A., Quesenberry, K., Unruh, B., Erikson, J., ... Munoz, P. (2017). Breeding apomictic bahiagrass (Paspalum notatum Flügge) with improved turf traits. Plant Breeding, 136, 253-260.

Sartor, M. E., Rebozzio, R. N., Quarin, C. L., \& Espinoza, F. (2013). Patterns of genetic diversity in natural populations of Paspalum agamic complexes. Plant Systematics and Evolution, 299, 1295-1306.

Tomas, M. A., Berone, G. D., Pisani, J. M., Ribotta, A. N., \& Biderbost, E. (2007). Relación entre el peso de semillas, poder germinativo y emergencia de plántulas en clones de Panicum coloratum L. Revista Argentina de Producción Animal, 27, 205-206.

Toutz, D. (1989). Hypervariability of simple sequences as a general source for polymorphic DNA markers. Nucleic Acids Research, 17, 6463-6471.

Urbani, M. H., Acuña, C. A., Doval, D. W., Sartor, M. E., Galdeano, F., Blount, A. R., ... Quarin, C. L. (2017). Registration of 'Boyero UNNE' Bahiagrass. Journal of Plant Registrations, 11, 26-32.

Vogel, K. P., \& Burson, B. L. (2004). Breeding and genetics. In L. E. Moser, et al. (Eds.), Warm-season (C4) grasses, Agronomy Monography 45 (pp. 51-94). Madison, WI: ASA, CSSA, SSSA.

West, S. H., \& Marousky, F. J. (1989). Mechanism of dormancy in Pensacola bahiagrass. Crop Science, 29, 787-791.

Williams, R. C., \& Webb, B. C. (1958). Seed moisture relationships and germination behavior of acid scarified bahiagrass seed. Agronomy Journal, 50, 235-237.

Zilli, A. L., Acuña, C. A., Schulz, R. R., Brugnoli, E. A., Guidalevich, V., Quarin, C. L., \& Martínez, E. J. (2018). Widening the gene pool of sexual tetraploid bahiagrass: Generation and reproductive characterization of a sexual synthetic tetraploid population. Crop Science, 58, 762-772.

Zilli, A. L., Brugnoli, E. A., Marcón, F., Billa, M. B., Rios, E. F., Martínez, E. J., \& Acuña, C. A. (2015). Heterosis and expressivity of apospory in tetraploid bahiagrass hybrids. Crop Science, 55, 1189-1201.

How to cite this article: Zilli AL, Acuña CA, Schulz RR, et al. Transference of natural diversity from the apomictic germplasm of Paspalum notatum to a sexual synthetic population. Ann Appl Biol. 2019;1-11. https://doi.org/10. 1111/aab.12507 